\title{
Qualidade pós-colheita de frutos de tomateiro submetidos a preparados em altas diluições
}

\author{
Tatiani A Modolon'; Pedro Boff²; Joatan M da Rosa ${ }^{1}$; Paulina Mariele R de Sousa ${ }^{1}$; David José Miquelluti ${ }^{1}$ \\ ${ }^{1}$ UDESC-CAV, Depto. Fitotecnia, Av. Luis de Camões 2090, 88520-000 Lages-SC; tatimodolon@hotmail.com; joatanmachado@bol. \\ com.br; paulinamariele@hotmail.com; a2djm@cav.udesc.br; ${ }^{2}$ EPAGRI, Lab. de Homeopatia e Saúde Vegetal, C. Postal 181, 88506-080 \\ Lages-SC; pboff@epagri.sc.gov.br
}

\begin{abstract}
RESUMO
Frutos de tomateiro são sensíveis ao manuseio e sua qualidade é afetada pelo sistema de cultivo e pelas práticas de conservação pós-colheita. O objetivo deste trabalho foi avaliar o efeito de preparados em altas diluições nos atributos indicadores da conservação pós-colheita de frutos de tomateiro. O trabalho consistiu em experimento a campo no ciclo produtivo 2009/2010 e ensaio de laboratório em pós-colheita. Os preparados avaliados, em aplicações a campo, foram os nosódios de tomateiro e de juá (Solanum aculeatissimum) nas dinamizações 12 e 24DH e os preparados homeopáticos Arnica montana e Sulphur nas dinamizações $12 \mathrm{DH}$ e 24DH. Frutos provenientes de tomateiro não tratados foram submetidos a imersão em preparações de Calcarea carbonica nas dinamizações 6, 12 e 24CH. O nosódio de juá 12 e 24DH aumentaram o teor de SS ( ${ }^{\circ}$ Brix), atributo que melhora a palatabilidade e a qualidade para a industrialização. Calcarea carbonica em tratamento pós-colheita não interferiu nos atributos químicos e físicos de frutos de tomate, embora Calcarea carbonica $24 \mathrm{CH}$ retardasse a formação de frutos tipo molho.
\end{abstract}

Palavras-chave: Solanum lycopersicum, tomate, conservação, agro-homeopatia.

\begin{abstract}
Post-harvest quality of tomato fruits treated with high dilution preparations

Tomato fruits are sensitive to handling and its quality is determined by the crop system and the post-harvest conservation practices. The aim of this work was to evaluate the influence of high dilution preparations in the post-harvest attributes of tomato fruits. The research consisted of one field experiment in the 2009/2010 crop cycle and laboratory assay in post-harvest. The high dilution preparations studied under field conditions were nosodes of tomato plant and Solanum aculeatissimum at 12 and 24DH (decimal hahnemanian dilutions) and the homeopathies Arnica montana and Sulphur at 12 and 24DH. In the post-harvest treatments, Calcarea carbonica was evaluated at 6,12 , and $24 \mathrm{CH}$ (centesimal hahnemanian dilution) fruits being deaped into the respective treatment. The nosode of $S$. aculeatissimum at 12 and $24 \mathrm{DH}$ increased the total content of soluble solids (Brix degree) which attribute enhances the flavor and quality for industrialization. Calcarea carbonica treatment in postharvest did not interfere on the chemical and physical attributes of tomato fruits, although Calcarea carbonica $24 \mathrm{CH}$ delayed the fruit softening.
\end{abstract}

Keywords: Solanum lycopersicum, tomato, conservation, agrohomeopathy.

(Recebido para publicação em 16 de dezembro de 2010; aceito em14 de dezembro de 2011) (Received on December 16, 2010; accepted on December 14, 2011)

$\mathrm{O}$ tomate é considerado um produto altamente perecível, demandando vários cuidados na sua conservação pós-colheita, dada a fragilidade dos seus tecidos e manutenção de sua atividade metabólica (Damasceno et al., 2003). Após formação dos frutos, o seu amadurecimento inicia-se com a elevação da atividade respiratória, típico de fruto climatério, acarretando transformações em suas características físicas e químicas, dentre elas a perda de clorofila, síntese de carotenoides e amolecimento dos tecidos (Vieites, 1998). Por outro lado, a aceitação dos frutos do tomateiro no mercado consumidor é notadamente considerada pela sua aparência e sabor. Isto indica que a avaliação de qualidade deva considerar os atributos físicos, sensoriais e a sua composição química, podendo ser expressa no conjunto de textura, açúcar e acidez (Borguini, 2006). Esses atributos são importantes não apenas para satisfazer as exigências do consumidor, mas como critérios de seleção genética de novas cultivares, seleção de práticas otimizadas de produção e de práticas adequadas ao manuseio pós-colheita (Chitarra \& Chitarra, 1990).

Técnicas de conservação de frutos pós-colheita têm sido preconizadas para a redução da atividade respiratória com o uso de películas comestíveis, aplicação de cera e armazenamento refrigerado. Recentemente, preparados em altas diluições têm demonstrado alta eficácia nos tratamentos fitossanitários de hortaliças, sem haver resíduos das plantas e efeitos negativos no meio ambiente. Preparados em altas diluições podem ser também potenciais indutores de mudanças fisiológicas, notadamente na produção de metabólitos secundários que afetam a conservação pós-colheita. Lisboa (2006) relata o aumento na assimilação de $\mathrm{CO}_{2}$ de plantas de arruda quando tratadas com o preparado Cantharis $4 \mathrm{CH}$ (4a ordem de diluição centesimal hahnemaniana). Por outro lado, Batirola da Silva (2006) observou que plantas de margaridinha tratadas com Sulphur na dinamização $6 \mathrm{CH}$ apresentaram acréscimo na taxa fotossintética. Bonato et al. (2009) verificaram aumento substancial do teor de óleo essencial em plantas de menta tratadas 
com Sulphur nas dinamizações 12, 24 e 30CH. Andrade (2000) verificou aumento de $77 \%$ do teor de cumarina em planta de chambá (Justicia pectoralis) tratadas com preparados em altas diluições de Arnica montana e Sulphur.

Relata-se ainda que preparados em altas diluições têm efeito imediato persistente e não linear na rota metabólica das plantas, o que nos leva a pensar de sua aplicabilidade em tratamentos pós-colheita, podendo ser correlacionados com o grau de dinamização do preparado (Bonato \& Silva, 2003).

O objetivo deste trabalho foi avaliar o efeito de tratamentos fitossanitários em altas diluições nos atributos indicadores da conservação pós-colheita de frutos de tomateiro.

\section{MATERIAL E MÉTODOS}

O trabalho consistiu em experimento a campo na EPAGRI em Lages (SC), no ciclo produtivo 2009/2010 e ensaio de conservação pós-colheita no laboratório de Homeopatia e Saúde Vegetal da EPAGRI, com análises realizadas no laboratório de Fisiologia e Pós-colheita da UDESC em Lages (SC).

$\mathrm{O}$ experimento a campo foi conduzido com tratamentos em pulverizações nas plantas com os seguintes preparados: nosódios de tomateiro (Solanum lycopersicum) e de juá (Solanum aculeatissimum) nas dinamizações 12 e $24 \mathrm{DH}\left(12^{\circ}\right.$ e $24^{\circ}$ ordem de diluição decimal hahnemaniana); preparados homeopáticos Arnica montana e Sulphur nas dinamizações 12 e 24DH; calda bordalesa a 0,3\%, calda cúprica EEC a 50 ppm (vinagre de uva, sementes de linho e sulfato de cobre) e testemunha sem intervenção. A dose dos preparados dispensada no pulverizador foi de $10 \mathrm{~mL} \mathrm{~L}^{-1}$. As pulverizações foram semanais realizadas com pulverizador costal manual com capacidade de $5 \mathrm{~L}$ $\left(\right.$ Guarany $^{\circledR}$ ) até a plena cobertura foliar, iniciadas aos 27 dias após o transplante e estenderam-se até 7 dias antes da última colheita. $\mathrm{O}$ delineamento experimental a campo foi de blocos casualizados com três repetições, sendo que a parcela experimental foi composta por 10 plantas de tomateiro com espaçamento de 0,50 $\mathrm{m}$ entre plantas dispostas em fileiras duplas espaçadas por $1 \mathrm{~m}$. Avaliações de $\mathrm{pH}$ e sólidos solúveis foram realizados a partir de cinco frutos inteiros e polpas de cinco frutos de cada parcela referente à primeira colheita (aos 70 dias após o transplante). Nas avaliações dos frutos inteiros, os mesmos foram triturados e homogeneizados em liquidificador. Amostras de polpas foram retiradas com auxílio de utensílios individuais para cada tratamento.

No ensaio em laboratório, frutos de tomate não tratados foram obtidos do campo experimental da EPAGRI de Lages (SC), selecionados quanto à uniformidade de tamanho e coloração (verde na escala 1 de acordo com CEAGESP, 2000) e transportados para o laboratório de Homeopatia e Saúde Vegetal. Os frutos passaram por desinfestação em solução de cloro ativo a $1,5 \%$ por 15 minutos. Após este período, os frutos foram lavados em água corrente oriunda de rede pública e em seguida em água deionizada e secos com papel toalha, para retirada de possíveis resíduos. $\mathrm{O}$ delineamento foi de blocos casualizados com quatro repetições, sendo que cada repetição foi composta por uma bandeja de plástico branca contendo dez frutos. Os tratamentos constituíram-se do preparado homeopático Calcarea carbonica nas dinamizações 6,12 e $24 \mathrm{CH}$ (escala de diluição decimal hahnemaniana) em etanol a 5\%, água deionizada e testemunha sem intervenção. Em cada tratamento utilizou-se a dose de $100 \mathrm{~mL}$ do preparado para 1,5 $\mathrm{L}$ de água deionizada, ficando os frutos mergulhados por 30 minutos. Após este período, os frutos foram retirados com ajuda de um escorredor, a bandeja foi seca individualmente com papel toalha e os frutos repostos no mesmo recipiente. $\mathrm{O}$ armazenamento dos frutos ocorreu em condições ambientes, com temperaturas médias de $20 \pm 5^{\circ} \mathrm{C}$, umidade relativa de $60 \pm 5 \%$ e intensidade luminosa de aproximadamente 10 horas em 410 lux e 14 horas em 0,01 lux.

Para ambos os experimentos, os valores de sólidos solúveis (SS), acidez titulável (AT) e pH foram determinados a partir de suco preparado com centrífuga (Britânia ${ }^{\circledR}$ mod. Turbo Juicer Prime). O teor de sólidos solúveis foi medido usando-se refratômetro digital (Atago ${ }^{\circledR} \bmod$ PR-201 $\alpha$ ) e o resultado expresso em graus brix. A acidez foi determinada pela titulação de $10 \mathrm{ml}$ de suco de tomate com $\mathrm{NaOH} 0,1 \mathrm{~N}$ até $\mathrm{pH} 8,2$, usando-se titulador automático (Schott ${ }^{\circledR}$ mod. Tritoline Easy) de acordo com Krammes et al. (2003). A acidez titulável foi expressa em percentagem, assumindo o ácido cítrico como ácido predominante no suco de tomate.

No ensaio em laboratório, as avaliações da perda de massa fresca e coloração da casca, foram realizadas aos $0,3,4,6,8,10,12$ e 14 dias de armazenamento após aplicação dos tratamentos. A perda de massa ao longo do armazenamento foi expressa em porcentagem, calculada sobre o peso inicial $\left(\mathrm{P}_{\text {inicial }}-\mathrm{P}_{\text {final }} / \mathrm{P}_{\text {inicial }} \times 100\right)$, utilizando uma balança com precisão de 0,01 $\mathrm{g}$ (Bel Engineering $^{\circledR}$ mod. Mark 500). A coloração da casca foi avaliada seguindo as Normas e Padrões de Classificação do Tomate da Companhia de Entrepostos e Armazéns Gerais de São Paulo (CEAGESP, 2000), com escala de 1 a 5, onde (1) Verde; (2) Salada; (3) Colorido; (4) Maduro; (5) Molho.

As avaliações de textura foram realizadas aos 14 dias de armazenamento quando $70 \%$ da testemunha atingiu nota de coloração 4 e 5 (CEAGESP, 2000). Os atributos de textura foram analisados com um texturômetro eletrônico (TAXT-plus ${ }^{\circledR}$ Mod. Stable Micro Syste$\mathrm{ms}$ ), em termos de força para ruptura da epiderme, para a penetração na polpa e para a compressão do fruto inteiro. Para a quantificação da força para ruptura da epiderme e para a penetração na polpa, foi utilizada uma ponteira, modelo PS2, com $2 \mathrm{~mm}$ de diâmetro, sem remoção da epiderme, a qual foi introduzida na polpa a uma profundidade de $5 \mathrm{~mm}$ com velocidades pré-teste, teste e pós-teste de 10,5 e $10 \mathrm{~mm} \mathrm{~s}^{-1}$, respectivamente. A resistência do fruto à compressão foi determinada usando-se uma plataforma plana, modelo $\mathrm{P} / 75$, com $75 \mathrm{~mm}$ de diâmetro, que exerceu uma força de compressão até uma deformação de 3 $\mathrm{mm}$ na superfície do fruto.

As análises estatísticas foram conduzidas conforme o delineamento experimental de blocos casualizados, adotando-se modelo linear de análise 
de variância. Foram adotadas análises univariadas para todas as variáveis com exceção da perda de massa. As comparações entre os valores médios, de cada uma das variáveis nos diferentes tratamentos, foram efetuadas por meio do teste de Tukey. A variável perda de massa foi submetida a análise de variância multivariada. Aos valores da variável perda de massa foi adicionada a constante $3 / 8$, elevados à potência $1 / 2 \mathrm{e}$ obtendo-se a seguir a função seno inversa (transformação arco-seno). Todas as transformações foram efetuadas conforme sugeridas pela análise descritiva dos dados. Os resultados são apresentados na escala original. Todas as análises foram executadas usando-se os procedimentos GLM (Littel et al., 1991) e MIXED (Littel et al., 2006) do software computacional SAS ${ }^{\circledR}$ (Versão 9.1). Para todos os testes efetuados foi considerado o nível mínimo de significância de 5\%.

\section{RESULTADOS E DISCUSSÃO}

Preparados em altas diluições, em escala centesimal ou decimal, não interferiram na acidez de frutos de tomate inteiros e nem de sua polpa, avaliados no estádio "maduro" (Tabela 1 e 2). A acidez é fator de reconhecida importância quando se analisa o nível de aceitação do produto no mercado (Borguini, 2006). Da mesma forma, a acidez é um fator de controle que regula muitas reações químicas e microbiológicas. Para o extrato de tomate, o $\mathrm{pH}$ indicado como ótimo é de 4,2 a 4,3, o qual impediria a proliferação de microrganismos e prolongaria o seu período de validade (Silva \& Giordano, 2000). Os valores de $\mathrm{pH}$ em nosso estudo foram semelhantes aos citados por Sobreira et al. (2010) e Liu et al. (2010). Da mesma forma, a acidez dos frutos tratados com preparados em altas diluições foi semelhante aos de frutos de tomateiro cultivado em sistema orgânico de produção observados por Arbos et al. (2010). De acordo com Chitarra \& Chitarra (1990), os ácidos orgânicos encontram-se dissolvidos nos vacúolos das células, tanto na forma livre, como combinada, formando sais, ésteres e glicosídeos. Em frutas, os ácidos orgânicos não só contribuem para a conservação como também para o sabor e aroma característico (Borguini, 2006).

Além da acidez, o sabor do fruto de tomate e da massa de tomate é determinado em grande parte pelo conteúdo de sólidos solúveis e de compostos voláteis (Sobreira et al., 2010). Em nosso trabalho, plantas de tomateiro tratadas com o nosódio de juá tiveram incremento no teor de sólidos solúveis nos frutos, chegando a $6,4{ }^{\circ}$ Brix no melhor tratamento. Vale ressaltar que, os valores de sólidos solúveis dos frutos tratados com o nosódio de juá nas dinamizações 6 e 12DH foram superiores aos observados por Sobreira et al. (2010) que obtiveram valor médio de 2,3 ${ }^{\circ}$ Brix em 18 acessos de tomate tipo Salada (Tabela 1). Nosso trabalho mostra ainda que os valores de sólidos solúveis nos frutos não tratados foram equivalentes aos observados por Borguini (2006), que obteve valores entre 4 e 4,5 para frutos da cultivar Carmem. Também, segundo estudo de Jones \& Scott (1984), a maior contribuição para o sabor e consequente aceitabilidade, é dada pela acidez e valores totais de açúcar encontrados nos frutos. Martinez-Valverde et al. (2002) avaliaram nove cultivares de tomate produzidos na Espanha, e registraram teores de sólidos solúveis entre 4 e 7,5 ${ }^{\circ}$ Brix. Segundo Borguini (2002), as matérias primas recebidas pelas indústrias no Brasil têm apresentado teor Brix em torno de 4,5, inferior ao relatado por Takeota et al. (2001), em que o teor de sólidos solúveis para as diversas cultivares de tomate varia entre 5 e $7^{\circ}$ Brix. Portanto, o tratamento de alta diluição de juá possibilita melhoria na qualidade da matéria prima a ser industrializada ou mesmo no consumo in natura.

Nos ensaios pós-colheita observou-se que os atributos de acidez e sólidos solúveis não foram afetados pelos tratamentos com os preparados em altas diluições de Calcarea carbonica em comparação com a testemunha. Frutos de tomate tratados com Calcarea carbonica apresentaram valores médios de acidez e de ácido cítrico de 4,21 e $0,40 \%$, respectivamente (Tabela 2 ). $\mathrm{O}$ preparado Calcarea carbonica é citado pelo efeito sobre a fisiologia do cálcio e poderia resultar na maior firmeza dos frutos de tomate, porem, esta tendência não foi confirmada em nosso estudo. (Vijnovsky, 1980). Plantas com distúrbio no metabolismo de cálcio e da relação fósforo-cálcio poderiam ser equilibradas com o uso do preparado Calcarea carbonica (Casali et al. 2009). O preparado Calcarea carbonica é citado também pelo efeito sobre os polifenóis de plantas medicinais, como observado por Fonseca et al. (2006)

Tabela 1. Valor de $\mathrm{pH}$ e teor de sólidos solúveis de frutos oriundos de plantas de tomateiro tratadas com preparados em altas diluições $(\mathrm{pH}$ value and soluble solids of tomato fruits harvested from plants treated with high dilution preparations). Lages, EPAGRI, 2010.

\begin{tabular}{lcccccc}
\hline \multirow{2}{*}{ Tratamentos } & \multicolumn{2}{c}{ Tomate inteiro } & & \multicolumn{2}{c}{ Polpa do tomate } \\
\cline { 2 - 3 } \cline { 6 - 7 } & $\mathbf{p H}$ & SS $\left({ }^{\circ}\right.$ Brix $)$ & & pH & SS ( ${ }^{\circ}$ Brix $)$ \\
\hline Nosódio de juá 12DH & $4,08^{\mathrm{ns}}$ & $6,36 \mathrm{a}$ & & $4,10^{\mathrm{ns}}$ & $6,20 \mathrm{a}$ \\
Nosódio de juá 24DH & 3,96 & $6,26 \mathrm{a}$ & & 4,03 & $6,40 \mathrm{a}$ \\
Calda cúprica EEC (50 ppm) & 4,06 & $4,50 \mathrm{~b}$ & & 4,08 & $4,66 \mathrm{~b}$ \\
Sulphur 12DH & 4,00 & $4,23 \mathrm{bc}$ & & 4,02 & $4,03 \mathrm{c}$ \\
Nosódio de tomateiro 12DH & 3,99 & $4,10 \mathrm{bc}$ & & 4,01 & $4,06 \mathrm{c}$ \\
Sem intervenção & 4,08 & $4,00 \mathrm{bc}$ & & 4,02 & $4,06 \mathrm{c}$ \\
Arnica montana 12DH & 4,04 & $4,00 \mathrm{bc}$ & & 4,08 & $4,00 \mathrm{c}$ \\
Nosódio de tomateiro 24DH & 3,96 & $3,93 \mathrm{bc}$ & & 3,98 & $3,93 \mathrm{c}$ \\
Sulphur 24DH & 3,97 & $3,90 \mathrm{bc}$ & & 3,97 & $4,00 \mathrm{c}$ \\
Arnica montana 24DH & 3,99 & $3,90 \mathrm{bc}$ & & 4,01 & $3,97 \mathrm{c}$ \\
Calda bordalesa (0,3\%) & 4,04 & $3,66 \mathrm{c}$ & & 3,98 & $4,00 \mathrm{c}$ \\
\hline CV (\%) & 2,64 & 8,91 & & 1,83 & 4,02 \\
\hline
\end{tabular}

Médias seguidas pela mesma letra, na coluna, não diferem entre si pelo teste de Tukey $(p \leq 0,05)$ (means followed by the same letter in the column did not differ from each other by Tukey test $(\mathrm{p} \leq 0.05)) .{ }^{\mathrm{ns}}=$ Não significativo pelo teste de de Tukey $(\mathrm{p} \leq 0,05)($ not significant by Tukey test $(\mathrm{p} \leq 0.05))$. 
Tabela 2. Conservação pós-colheita de frutos de tomate tratados com preparados em altas diluições de Calcarea carbonica (post-harvest conservation of tomato fruits treated by high dilutions of Calcarea carbonica). Lages, EPAGRI, 2010.

\begin{tabular}{|c|c|c|c|c|c|c|}
\hline \multirow{2}{*}{ Tratamentos } & \multicolumn{3}{|c|}{ Atributos químicos $^{1}$} & \multicolumn{3}{|c|}{ Atributos de firmeza (N) } \\
\hline & SS & AT & pH & Casca $^{2}$ & Polpa $^{3}$ & Compressão $^{4}$ \\
\hline Calcarea carbonica $12 \mathrm{CH}$ & $3,97^{\mathrm{ns}}$ & $0,38^{\mathrm{ns}}$ & $4,55^{\mathrm{ns}}$ & $6,22^{\mathrm{ns}}$ & $1,11^{\mathrm{ns}}$ & $8,52^{\mathrm{ns}}$ \\
\hline Sem intervenção & 4,30 & 0,39 & 4,10 & 6,21 & 0,76 & 9,06 \\
\hline Água deionizada & 4,17 & 0,39 & 4,21 & 6,09 & 0,90 & 8,68 \\
\hline Calcarea carbonica $6 \mathrm{CH}$ & 4,15 & 0,40 & 4,12 & 6,25 & 1,05 & 8,33 \\
\hline Calcarea carbonica $24 \mathrm{CH}$ & 4,12 & 0,43 & 4,04 & 6,16 & 1,15 & 8,23 \\
\hline CV (\%) & 5,39 & 8,06 & 7,12 & 6,52 & 37,81 & 9,70 \\
\hline
\end{tabular}

${ }^{\mathrm{ns}}=$ Não significativo pelo teste de Tukey $(\mathrm{p} \leq 0,05)$ (not significant by Tukey test $\left.(\mathrm{p} \leq 0.05)\right) ;{ }^{\mathrm{S} S}=$ Sólidos solúveis expresso em ${ }^{\circ}$ Brix $($ soluble solids expressed by the Brix degree), AT = Acidez titulável expressa em percentual de ácido cítrico (acidity expressed by the percentage of citric acid). ${ }^{2}=$ Força para ruptura da casca (power to break the peal). ${ }^{3}=$ Força para penetração da polpa (power for penetration inside the fruit). ${ }^{4}=$ Resistência do fruto a compressão (fruit resistance against pressure).

Tabela 3. Perda de massa de frutos submetidos a tratamento pós-colheita com preparados em altas diluições (fruit weight losses after fruit treatment with high dilution preparations at post-harvest conditions). Lages, EPAGRI, 2010.

\begin{tabular}{lccccccc}
\hline \multirow{2}{*}{ Tratamentos } & \multicolumn{7}{c}{ Perda de massa fresca (\% por fruto) } \\
\cline { 2 - 8 } & $* \mathbf{3}$ & $\mathbf{4}$ & $\mathbf{6}$ & $\mathbf{8}$ & $\mathbf{1 0}$ & $\mathbf{1 2}$ & $\mathbf{1 4}$ \\
\hline Calcarea carbonica $12 \mathrm{CH}$ & $1,4^{\mathrm{ns}}$ & $3,5^{\mathrm{ns}}$ & $6,3^{\mathrm{ns}}$ & $7,4^{\mathrm{ns}}$ & $8,1^{\mathrm{ns}}$ & $8,8^{\mathrm{ns}}$ & $9,5^{\mathrm{ns}}$ \\
Sem intervenção & 1,7 & 2,3 & 3,3 & 3,7 & 4,8 & 5,5 & 6,0 \\
Água deionizada & 1,4 & 2,0 & 3,2 & 3,6 & 5,3 & 5,8 & 6,5 \\
Calcarea carbonica $6 \mathrm{CH}$ & 1,4 & 2,2 & 3,4 & 4,0 & 7,0 & 7,7 & 8,4 \\
Calcarea carbonica $24 \mathrm{CH}$ & 1,3 & 1,9 & 3,0 & 3,6 & 7,6 & 11,2 & 8,3 \\
\hline
\end{tabular}

*Dias após a instalação do experimento (days after installing the experiment); ${ }^{\text {ns }}=$ Não significativo pelo teste de Tukey $(\mathrm{p} \leq 0,05)$ (not significant by Tukey test $(\mathrm{p} \leq 0.05)$ ).

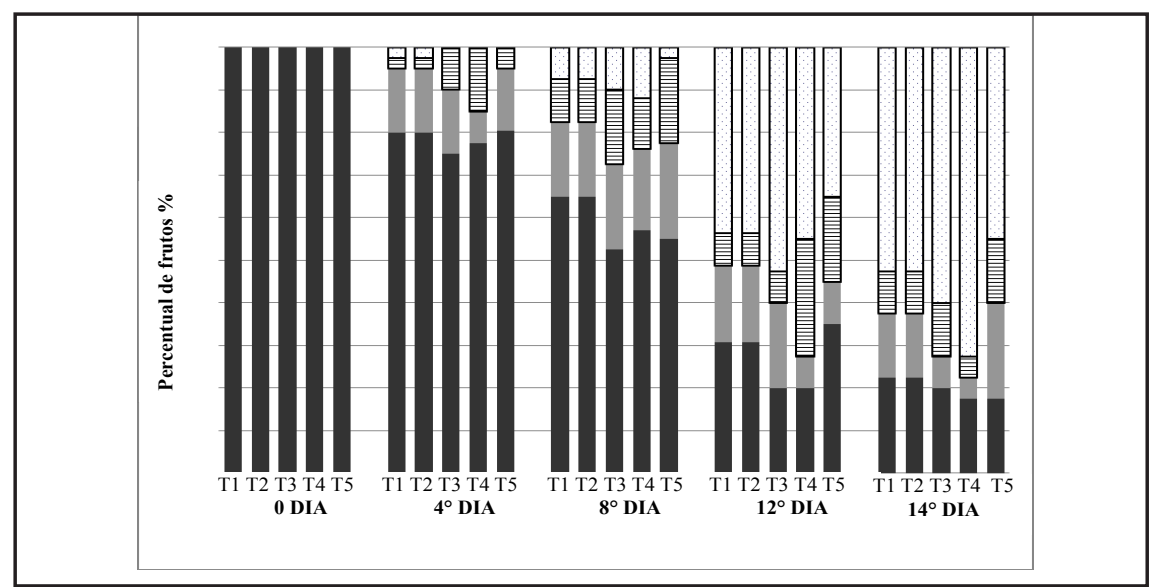

Figura 1. Maturação de frutos de tomateiro submetidos a tratamento com preparados em altas diluições de Calcarea carbonica (ripening tomato fruits treated with Calcarea carbonica at high dilution preparations). Lages, EPAGRI, 2010.

$\mathrm{T} 1$ = Calcarea carbonica $12 \mathrm{CH} ; \mathrm{T} 2=$ Sem intervenção; $\mathrm{T} 3=$ Água deionizada; $\mathrm{T} 4=$ Calcarea carbonica $6 \mathrm{CH}$; T5 = Calcarea carbonica 24CH; Verde (escala 1); Salada e colorido (escala 2 e 3); Maduro (escala 4); Molho (escala 5) (CEAGESP, 2000).

que verificaram incremento do teor de tanino em plantas de couve-cravinho (Porophyllum ruderale) com a aplicação única de Calcarea carbonica 4CH.

Chiumarelli \& Ferreira (2006), estudando tratamento pós-colheita de so et al. (2006) que também estudaram a cultivar 'Santa Cruz Kada' e obtiveram valor médio de $\mathrm{pH}$ de 4,35 com 3,95 de ${ }^{\circ}$ Brix. Caliman (2003), por outro lado, obteve $0,30 \%$ de ácido cítrico em frutos de tomate cultivados a campo e em ambiente protegido, índices inferiores ao obtidos em nosso estudo.

A perda de firmeza da polpa é uma característica comum que ocorre durante o amadurecimento dos frutos e é muito importante do ponto de vista econômico, já que afeta a qualidade e a resistência dos produtos ao transporte e apodrecimento (Awad, 1993). Em nosso estudo, a firmeza dos frutos avaliada pela força necessária para romper a casca e polpa e resistência à compressão não foram afetadas pelos tratamentos com Calcarea carbonica (Tabela 2). Isto pode ser confirmado também com a perda de massa fresca, embora a perda de massa tenha sido maior na testemunha sem intervenção no terceiro dia após início do ensaio (Tabela 3). A alteração na firmeza da polpa pode ser atribuída a dois processos: a perda excessiva de água dos tecidos, que causa diminuição da pressão de turgor, comum em situação de armazenamento em baixa umidade relativa do ar e às modificações observadas na lamela média e parede celular, principalmente devido à atividade enzimática (Kluge et al. 1997). Nossos resultados de perda de massa fresca são semelhantes aos encontrados por Chiumarelli \& Ferreira (2006), avaliando coberturas comestíveis na pós-colheita do tomate à temperatura de $25^{\circ} \mathrm{C}$. Estes autores observaram percentuais de perda de massa entre 0,48 e 1,10 no terceiro dia, 
e 1,61 e 4,05 no nono dia após serem submetidos aos tratamentos com cera e fécula de mandioca. Por outro lado, os valores de perda de massa fresca encontrados em nosso estudo no décimo dia após a aplicação dos tratamentos foram superiores aos observados por Andreuccetti et al. (2007). Esses autores obtiveram em média $3 \%$ de perda de massa fresca em tomates armazenados em temperatura de 12,5 e $20^{\circ} \mathrm{C}$ e com uso de etileno, enquanto que no nosso estudo foi entre 4,8 e 8,1\% (Tabela 3). Isso indica que a atividade de etileno na promoção de alterações metabólicas dos tecidos vegetais dos frutos de tomate não foi afetada pelos preparados em altas diluições.

Pode-se dizer que frutos oriundos de plantas de tomateiros tratadas com nosódio de juá têm potencial de melhor palatabilidade e qualidade para industrialização, já que quanto maior o teor de SST ( ${ }^{\circ}$ Brix) maior será o rendimento em nível industrial (Monteiro et al. 2008).

A maturação de frutos de tomateiro não foi afetada pelos tratamentos pós-colheita de altas diluições de Calcarea carbonica até o $14^{\circ}$ dia de armazenamento (Figura 1). Entretanto, Calcarea carbonica na dinamização $24 \mathrm{CH}$ parece ter retardado a proporção de frutos para molho, apresentando maior percentual de frutos do tipo salada e colorido cerca de $25 \%$ (Figura 1). O preparado em alta diluição de Calcarea carbonica $24 \mathrm{CH}$ pode ter diminuído a produção de etileno dos frutos fazendo com que estes demorassem mais tempo para chegar ao tipo maduro e molho. A cor é o atributo de qualidade que serve de indicativo direto para o consumidor. Os consumidores que preferem frutos de tomate do tipo salada e coloridos, mas não do tipo molho, seriam melhor atendidos com processos que retardassem o amadurecimento, mas permitissem cor próxima ao vermelho. Por outro lado, a proporção de frutos verdes aproxima-se em todos os tratamentos a 20\% no final deste período. Ao contrário, Calcarea carbonica na dinamização $6 \mathrm{CH}$ parece ter acelerado o amadurecimento dos frutos tratados pós-colheita com este preparado. Tomates do tipo molho são perecíveis e muito suscetíveis a danos durante a comercialização, portanto, não são resistentes ao rigor do sistema de manuseio pós-colheita (Moura et al. 1999). Após a colheita dos frutos climatéricos, a respiração torna-se o seu principal processo fisiológico. Neste período, os frutos passam a utilizar suas próprias reservas para continuar o seu desenvolvimento, porém a energia liberada pela respiração pode ser utilizada, em alguns casos, para continuar a síntese de pigmentos, enzimas e outros materiais de estrutura molecular elaborada (Chitarra \& Chitarra, 1990). Neste sentido, o preparado Calcarea carbonica $6 \mathrm{CH}$ parece ter acelerado a respiração ou favorecido a assimilação da energia liberada na respiração para a síntese de pigmentos, antecipando o amadurecimento dos frutos. Este resultado, aparentemente contrastante entre dois níveis de dinamização (6 e 24CH) para o mesmo preparado, tem sido frequentemente observada em preparados em altas diluições, resultando em efeito oscilatório com o aumento da dinamização do preparado, como argumentado por Bonato et al. (2009), ao invés do esperado efeito linear, comum em doses ponderais.

\section{AGRADECIMENTOS}

Ao MCT/CT-HIDRO/CNPq através do projeto Rede Guarani/Serra Geral convênio FAPEU/FAPESC 16261/102 e FAPESC convênio 7025/2010-4 pelo apoio financeiro para condução da pesquisa. A primeira autora agradece à CAPES pela concessão da bolsa de mestrado.

\section{REFERÊNCIAS}

ANDRADE FMC. 2000. Homeopatia no crescimento e produção de cumarina em chambá Justicia pectoralis Jacq. Viçosa: UFV. 124p. (Dissertação mestrado).

ANDREUCCETTI C; FERREIRA MD MORETTI CL; HONÓRIO SL. 2007. Qualidade pós-colheita de frutos de tomate cv. Andréa tratados com etileno. Horticultura Brasileira 25: 122-126.

ARBOS KA; FREITAS RJS; STERTZ SC; CARVALHO LA. 2010. Segurança alimentar de hortaliças orgânicas: aspectos sanitários e nutricionais. Ciência e Tecnologia de Alimentos 30: 215-220.

AWAD M. 1993. Fisiologia pós-colheita de frutos. São Paulo: Nobel. 114p.

BATIROLA DA SILVA RT. 2006. Interpretação matemático-fisica dos efeitos de ulttradiluições em Sphagneticola trilobata (L.) Pruski. Viçosa: UFV. 127p (Dissertação mestrado).

BONATO CM; SILVA EP. 2003. Effect of the homeopathic solution Sulphur on the growth and productivity of radish. Acta Scientiarum. Agronomy 25: 259-263.

BONATO CM; PROENÇA GT; REIS B. 2009. Homeopathic drugs Arsenicum album and Sulphur affect the growth and essential oil content in mint (Mentha arvensis L.). Acta Scientiarum. 31: 101-105.

BORGUINI RG. 2006. Avaliação do potencial antioxidante e de algumas características físico-químicas do tomate (Lycopersicon esculentum) orgânico em comparação ao convencional. São Paulo: USP-FSP. 161p. (Tese doutorado).

BORGUINI RG. 2002. Tomate (Lycopersicon esculentum Mill.) orgânico: o conteúdo nutricional e a opinião do consumidor. Piracicaba: USP-ESALQ. 110p. (Dissertação mestrado).

CALIMAN FRB. 2003. Produção e qualidade de frutos de genótipos de tomateiro em ambiente protegido e no campo. Viçosa: UFV. $72 \mathrm{p}$. (Dissertação mestrado).

CARDOSO SC; SOARES ACF; BRITO AS; CARVALHO LA; PEIXOTO CC; PEREIRA MEC; GOES E. 2006. Qualidade de frutos de tomateiro com e sem enxertia. Bragantia: 65: 269-274.

CASALI VWD; ANDRADE FMC; DUARTE ESM. 2009. Acologia de altas diluições. Viçosa: UFV-Departamento de Fitotecnia. $537 \mathrm{p}$.

CEAGESP (Companhia de Entrepostos e Armazéns Gerais do Estado de São Paulo). 2000. Classificação de Tomate. Programa Horti\&Fruti, $3 \mathrm{p}$.

CHITARRA MIF; CHITARRA AB. 1990. Póscolheita de frutos e hortaliças: Fisiologia e manuseio. Lavras: ESALQ-FAEPE. 320p.

CHIUMARELLI M; FERREIRA MD. 2006. Qualidade pós-colheita de tomates 'Débora' com utilização de diferentes coberturas comestíveis e temperaturas de armazenamento. Horticultura brasileira 24: 381-385.

DAMASCENO S; OLIVEIRA PVS; MORO E; MACEDO JUNIOR EK; LOPES MC; VICENTINI NM. 2003. Efeito da aplicação de película de fécula de mandioca na conservação pós-colheita de tomate. Ciência e Tecnologia de Alimentos 23: 377-380.

FONSECA MCM; CASALI VWD; CECON PR. 2006. Efeito de aplicação única dos preparados homeopáticos Calcarea carbonica, Kalium phosphoricum, Magnesium carbonicum, Natricum muriaticum e Silicea terra no teor de tânico em Porophyllum ruderale (Jacq.) Cassini. Cultura Homeopática 4:6-8.

JONES RA; SCOTT SJ. 1984. Genetic potential to improve tomato flavor in F[1] hybrids. Journal of American Society for Horticultural Science 109: 318-321.

LISBOA SP. 2006. Antagonismo de preparações homeopáticas na fotossintese de plantas de Ruta graveolens (L.). Viçosa: UFV. 103p (Dissertação mestrado).

LITTEL RC; FREUND RJ; SPECTOR PC. 1991. 
SAS System for Linear Models. Cary, NC, USA: SAS Institute Inc. $3^{\mathrm{a}}$ edição, 329 p.

LITTEL RC; MILLIKEN GA; STROUP WW; WOLFINGER RD; SCHABENBERGER O. 2006. SAS ${ }^{\circledR}$ for Mixed Models. SAS Institute Inc.: Cary, NC, USA. 834p.

LIU F; CAO X; WANG H; LIAO X. 2010. Changes of tomato powder qualities during storage. Powder Technology 204: 159-166.

MARTINEZ-VALVERDE I; PERIAGO MJ; PROVAN G. 2002. Phenolic compounds, lycopene and antioxidant activity in commercial varieties of tomato (Lycopersicum esculentum). Journal of the Science of Food and Agriculture 82: 323-330.

MOURA ML; SARGENT SA; OLIVEIRA RF. 1999. Efeito da atmosfera controlada na conservação de tomates colhidos em estádio intermediário de maturidade. Scientia Agricola
56: $135-142$.

KLUGE RA; NACHTIGAL JC; FACHINELLO JC; BILHALVAAJ. 1997. Fisiologia e manejo pós-colheita de frutas de clima temperado. Pelotas: UFPEL. $163 \mathrm{p}$.

KRAMMES JG; MEGGUER CA; ARGENTA LC; AMARANTE CVT; GROSSI D. 2003. Uso do 1-metilciclopropeno para retardar a maturação de tomate. Horticultura Brasileira. 21: 611-614.

MONTEIRO CS; BALBI ME; MIGUEL OG; PENTEADO PTPS; HARACEMIV SMC. 2008. Qualidade nutricional de antioxidante do tomate "Tipo Italiano". Alimentos e Nutrição 19: $25-31$

SILVA JBC; GIORDANO LB (org). 2000. Tomate para Processamento Industrial. Brasília: EMBRAPA-CNPH. 169p.

SOBREIRA FM; SOBREIRA FM; ALMEIDA
GD; COELHO RI; RODRIGUES R; MATTA FP. 2010. Qualidade de sabor de tomates dos tipos salada e cereja e sua relação com caracteres morfoagronômicos dos frutos. Ciência e Agrotecnologia 34: 015-1023.

STATISTICAL ANALYSIS SYSTEM, Institute $\operatorname{Inc}^{\circledR}$ 2003. SAS Ver. 9.1 3 SAS Institute Inc.: Cary, NC, USA. Lic. UDESC.

VIEITES RL. 1998. Conservação pós-colheita de tomate (Lycopersicum esculentum Mill). Botucatu: Fepaf.

TAKEOTA GR; DAO L; FLESSA S; GILLESPIE DM; JEWELL WT; HUEBNER B; BERTOW D; EBELER SE. 2001. Processing effects on lycopene content and antioxidant activity of tomatoes. Journal of the Science of Food and Agriculture 49: 3713-3717.

VIJNOVSKY B. 1980. Tratado de matéria médica homeopática. v1. Rio de Janeiro: Editora: Mukunda. 790p. 